\title{
A Loss or a Gain, Is It Not All the Same?
}

The human genome contains numerous loci of which losses or gains provoke clinical phenotypes, albeit often of quite a different nature. Apparently, the genes within these loci have to exist in exactly 2 copies. If there are 3 copies, due to a duplication, or only 1 copy, after a deletion, genes within the locus may exert 'dosage-sensitive' effects. This can only be the case if both alleles are being transcribed. If transcripts and proteins of only one copy were to be produced, this would result in haploinsufficiency. Also in the reciprocal situation, in which the cell transcribes 3 copies, transcript imbalance may arise. The gene dosage balance hypothesis provides a molecular framework for how genes encoding transcription factors and proteins involved in signal transduction and macromolecular complexes in general may cause clinical phenotypes [Veitia and Birchler, 2010; Poot et al., 2011].

Disorders resulting from losses or gains of dosage-sensitive genes often show remarkable phenotypic variability. Within the region 7q11.23, a loss causes WilliamsBeuren syndrome (WBS; OMIM 194050) and a duplication results in the 7q11.23 duplication syndrome (Dup7; OMIM 609757) [Osborne and Mervis, 2007]. The WBS is characterized by mild to moderate intellectual disability or learning difficulties, with relative cognitive abilities in verbal short-term memory and in language use but weaknesses in visuospatial construction, anxiety, attention deficit hyperactivity disorder, and social disinhibition. Patients with Dup7 syndrome show severely delayed speech and expressive language acquisition but relative good visuospatial functioning. A common inversion within the 7q11.23 region, WBSinv-1, exists as a poly-

\section{KARGER}

E-Mail karger@karger.com

www.karger.com/msy morphism in the general population and provokes no phenotype [Tam et al., 2008]. This indicates that severance of the WBS locus within 7q11.23 by a genomic rearrangement would not disrupt a putative transcription-associated domain [Spielmann and Klopocki, 2013; Spielmann and Mundlos, 2013; Poot and Haaf, 2015].

Being flanked by blocks of inverted low copy repeats, the WBS locus within the 7q11.23 region often gives rise to ring(7) chromosomes [Velagaleti et al., 2002]. Patients with a supernumerary ring(7) share speech difficulties, irrespective of whether the ring(7) is present in $100 \%$ of the cells or as a high- or low-grade mosaicism [Lichtenbelt et al., 2005]. For a girl with a ring(7) between 12 and $22 \mathrm{Mb}$ in size and present in 50\% of lymphocytes, the authors searched for potential candidate genes that may account for her problems with speech acquisition. Bearing in mind that candidate genes should be dosage-sensitive, they pinpointed 2 genes: STX1A and LIMK1. This reasoning was supported by functional data. The STX1A geneencoded syntaxin protein mediates vesicle docking, fusion, and neurotransmitter release. Overexpression of STX1A reduces the nerve growth factor-induced neurite extension in vitro [Zhou et al., 2000]. The LIMK1 and $L I M K 2$ genes encode kinases that regulate actin dynamics by phosphorylating cofilin and thus affect neuronal function, neurodevelopment and synaptic plasticity [Cuberos et al., 2015]. Given that this patient carried a 50\% mosaicism for ring(7), an increase of only $25 \%$ of syntaxin protein and/or LIMK1-encoded kinase activity would be sufficient to account for her clinical phenotypes [Lichtenbelt et al., 2005]. (c) 2016 S. Karger AG Basel

$1661-8769 / 16 / 0071-0001 \$ 39.50 / 0$ 
Another patient with a duplication of the WBS locus in 7 q11.23 showed moderate to severe language delay at 2 years of age, which worsened to a severe delay in receptive and expressive language at 3 years and 2 months. At 4 years and 2 months, he was diagnosed with attention deficit hyperactivity disorder, overall developmental delay, an unspecified sleep disorder, and a severe delay in speech and language and in fine motor skills [Somerville et al., 2005]. Gene expression analysis in lymphoblastoid cell lines by real-time PCR demonstrated increased expression for 5 of the 6 genes within the duplicated region that were examined (GTF2I, LIMK1, WBSCR1, RFC2, and $B A Z 1 B$ ). Thus, these data lend support to the previous suggestion of Lichtenbelt et al. [2005] and highlight possible deleterious effects of an even small increase in level of LIMK1 transcripts.

While most case reports of the WBS and Dup7 syndrome follow the gene-centered paradigm, a recent study took a novel approach to elucidate the effects of reciprocal losses and gains in the genome [Poot and Hochstenbach, 2010; Strong et al., 2015]. They noted that the dup7 locus harbors genes such as WBSCR22, which contains an Sadenosyl-L-methionine-binding domain typical of methyltransferases; NSUN5, which acts as an RNA-methyltransferase; $B A Z 1 B$, a subunit of the WICH and B-WICH chromatin remodeling complexes; $B C L 7 B$, being identified as a mammalian SWI/SNF ATP-dependent subunit of the chromatin-remodeling complex, and the transcrip- tion factors GTF2I and GTF2IRD1, which functionally interact with the histone deacetylase HDAC3. This prompted the authors to compare the effects of gene dosage in the region $7 \mathrm{q} 11.23$ on genome-wide DNA methylation of whole blood samples from 20 children with WBS, 10 with Dup7 syndrome and 20 samples from age-matched typically developing control children [Strong et al., 2015]. They found gene dose-dependent changes in DNA methylation in genes which were enriched for Gene Ontology categories involved in neurological development, function and disease, albeit that their analysis was done on whole blood-derived DNA. These data suggest that altered DNA methylation and consequential altered gene expression may affect the neurodevelopment in both WBS and Dup7 syndrome. This may, in turn, provoke distinct phenotypes, in particular some of the features of intellectual delay, autism spectrum, speech development, and anxiety disorders. During a decade of study, the WBS/ $\operatorname{dup}(7)$ locus in the $7 \mathrm{q} 11.23$ region has proved a rich source of information regarding developmental disorders, in particular of speech acquisition. In addition, our insights into how changes in DNA copy number may cause clinical phenotypes have progressed from a strictly genecentered paradigm to a multifaceted approach to the possible modes of gene action. Even 'simple' genome rearrangements such as losses and gains prove now 'complex' in their mechanisms of action and their eventual outcome [Poot and Hochstenbach, 2010; Poot and Haaf, 2015].

Martin Poot

\section{References}

Cuberos H, Vallée B, Vourc'h P, Tastet J, Andres CR, Bénédetti H: Roles of LIM kinases in central nervous system function and dysfunction. FEBS Lett 589:3795-3806 (2015).

Lichtenbelt KD, Hochstenbach R, van Dam WM, Eleveld MJ, Poot M, Beemer FA: Supernumerary ring chromosome 7 mosaicism: case report, investigation of the gene content, and delineation of the phenotype. Am J Med Genet A 132A:93-100 (2005).

Osborne LR, Mervis CB: Rearrangements of the Williams-Beuren syndrome locus: molecular basis and implications for speech and language development. Expert Rev Mol Med 9: 1-16 (2007).

Poot M, Haaf T: Mechanisms of origin, phenotypic effects and diagnostic implications of complex chromosome rearrangements. Mol Syndromol 6:109-133 (2015).
Poot M, Hochstenbach R: A three-step workflow procedure for the interpretation of arraybased comparative genome hybridization results in patients with idiopathic mental retardation and congenital anomalies. Genet Med 12:478-485 (2010).

Poot M, van der Smagt JJ, Brilstra EH, Bourgeron $\mathrm{T}$ : Disentangling the myriad genomics of complex disorders, specifically focusing on autism, epilepsy, and schizophrenia. Cytogenet Genome Res 135:228-240 (2011).

-Somerville MJ, Mervis CB, Young EJ, Seo EJ, del Campo M, et al: Severe expressive-language delay related to duplication of the WilliamsBeuren locus. N Engl J Med 353:1694-1701 (2005).

Spielmann M, Klopocki E: CNVs of noncoding cis-regulatory elements in human disease. Curr Opin Genet Dev 23:249-256 (2013).

-Spielmann M, Mundlos S: Structural variations, the regulatory landscape of the genome and their alteration in human disease. Bioessays 35:533-543 (2013).
Strong E, Butcher DT, Singhania R, Mervis CB, Morris CA, et al: Symmetrical dose-dependent DNA-methylation profiles in children with deletion or duplication of 7q11.23. Am J Hum Genet 97:216-227 (2015).

Tam E, Young EJ, Morris CA, Marshall CR, Loo $\mathrm{W}$, et al: The common inversion of the Williams-Beuren syndrome region at $7 \mathrm{q} 11.23$ does not cause clinical symptoms. Am J Med Genet A 146A:1797-1806 (2008).

-Veitia RA, Birchler JA: Dominance and gene dosage balance in health and disease: why levels matter! J Pathol 220:174-185 (2010).

-Velagaleti GV, Jalal SM, Kukolich MK, Lockhart LH, Tonk VS: De novo supernumerary ring chromosome 7: first report of a non-mosaic patient and review of the literature. Clin Genet 61:202-206 (2002).

Zhou Q, Xiao J, Liu Y: Participation of syntaxin $1 \mathrm{~A}$ in membrane trafficking involving neurite elongation and membrane expansion. J Neurosci Res 61:321-328 (2000). 